Article

\title{
An Analytical Cost-Optimal Cloudlet Placement Framework over Fiber-Wireless Networks with Quasi-Convex Latency Constraint
}

\author{
Sourav Mondal ${ }^{1, *(\mathbb{D})}$, Goutam Das ${ }^{2}$ and Elaine Wong ${ }^{1}$ (i) \\ 1 Department of Electrical and Electronic Engineering, The University of Melbourne, Melbourne, VIC 3010, \\ Australia; ewon@unimelb.edu.au \\ 2 G. S. Sanyal School of Telecommunications, Indian Institute of Technology Kharagpur, Kharagpur 721302, \\ India; gdas@gssst.iitkgp.ac.in \\ * Correspondence: smondal@student.unimelb.edu.au
}

Received: 15 March 2019; Accepted: 3 April 2019; Published: 5 April 2019

\begin{abstract}
To meet the low-latency constraints arising from future smart mobile devices, Internet-of-Things, and 5G applications, major interest is currently given to the integration of centralized cloud computing and distributed edge computing infrastructures to deliver higher performance and reliability to edge devices in accessing mobile cloud services. The three-tier network architecture arising from cloud, cloudlet, and edge-devices can handle miscellaneous latency requirements for both latency-sensitive and latency-tolerant applications more efficiently than conventional two-tier networks. In this paper, we primarily focus on the static cloudlet network planning problem and propose an analytical hybrid cost-optimization framework for optimal cloudlet placement. We formulate this problem as a convex optimization problem and solve by using Karush-Kuhn-Tucker (KKT) conditions, and show that this framework can be evaluated without any scalability issues observed with integer programming based frameworks for large datasets. Moreover, we derive user-friendly closed form expressions that provide a first-hand estimation of cloudlet deployment cost depending on a few important network parameters like split-ratio, population density, and network bandwidth. Finally, we also show that the optimal solution of this analytical framework can be considered as a tight lower bound of the optimal solutions of integer programming based frameworks and makes a better cloudlet installation cost estimation compared to other existing frameworks.
\end{abstract}

Keywords: cloudlet; cost-minimization; edge computing; fiber-wireless access network; quasi-convex optimization

\section{Introduction}

The recent advancements in mobile hardware technology have not only resulted in trendy, lightweight, and easily portable smart devices from bulky with limited functionality devices, but also paved the path for applications like augmented reality, cognitive assistance, and face recognition, to name a few. However, these applications are highly computation intensive, and this limited computing capability of portable mobile devices like iPhones, Android phones, and Google glasses are unable to support these applications. Although mobile cloud computing enables mobile devices to access a shared pool of configurable computational and storage resources, providing ubiquitous, convenient, and on demand services, large communication latency between mobile devices and remote clouds still presents a new challenge for low-latency applications that demand 1-100 ms end-to-end system latency [1]. To achieve this low-latency requirement, the authors of [2] proposed the idea 
of cloudlet computing similar to fog and mobile-edge computing, where cloudlets are considered as trusted, resource-rich computer or cluster of computers. Nonetheless, cloudlets are designed to be self-managing and function with low-context-awareness. Hence, they are most suitable for deployment in adversarial environments [3].

The authors of [4] showed that the system performance in terms of expected latency for the requested jobs from a single-hop cloudlet server is always better than a distant cloud server for low-latency applications. Thus, the optimal placement of cloudlets over wireless access networks seemed to be an important research problem to enhance the system performance. Along with the wireless access networks, researchers started to focus on optical and fiber-wireless (FiWi) access networks for edge-computing solutions deployment due to their wide deployment coverage, low cost per bit, very high data transmission bandwidth, efficient network virtualization and network scalability [5]. The time-division multiplexed passive optical network (TDM-PON) has also been considered as a front/back-haul support for wireless access network, i.e., access technologies like Radio-over-Fiber (RoF), WiFi, LTE-A and millimeter-wave (mmWave) can be integrated with optical network units (ONUs) for a higher user coverage [6].

A static cloudlet placement framework considers that the network is static in time, i.e., it does not consider user mobility and virtual machine (VM) mobility into account, and identifies optimal cloudlet locations over an existing access network infrastructure [7]. Nonetheless, most of the existing research on optimal cloudlet placement formulated integer/mixed-integer linear/nonlinear programming problems, which are NP-Hard problems. Hence, authors either relied on commercial solvers or designed heuristic algorithms, e.g., we proposed a mixed-integer nonlinear programming (MINLP) based hybrid cloudlet placement cost-optimization framework over TDM-PON based FiWi networks in [8] that relies on commercial solvers. However, both these approaches either suffer from scalability issues or from a strong bias arising from the particular dataset used for the framework evaluation. Apart from the computational aspects, these works do not provide any general insight on the behavior of the frameworks against variation in network parameters. However, these cloudlet placement frameworks provide exact cloudlet placement locations, but an existing network infrastructure is required as an input to these frameworks. Thus, when some network service provider intends to install cloudlet servers while deploying green-field fibers, an estimation of the cloudlet deployment cost appears to be really useful, but these existing cloudlet placement frameworks fail to do so, as there is no existing network infrastructure.

To address this gap in existing literature, in this paper, we propose an analytical cost-optimization framework for the hybrid cloudlet placement based on the assumption that the underlying access network is homogeneous, i.e., the same split ratio of each TDM-PON and the same number of users are served by each optical network unit (ONU), and the average distance between all ONUs and field, remote node (RN) and central office (CO) cloudlets are, respectively, equal. This analytical cost-optimization framework diminishes the computational complexity of the MINLP based framework proposed in [8] to a great extent by reducing the entire volume of network to a single TDM-PON based FiWi branch and provides a quick lower bound of cloudlet deployment cost. Note that we can directly convert the integer variables of the MINLP formulation in [8] as continuous variables and formulate a continuous convex optimization problem, which can provide a lower bound to the actual cloudlet installation cost. However, at this point, we ask the question "can we find a better lower bound for cloudlet installation cost?", i.e., if it is possible to find a framework that can find a tighter lower bound for the cloudlet deployment cost. In this context, we feel that directly using continuous variables to denote cloudlet installation decisions is not very appropriate and hence propose an alternative method. Our primary contributions in this paper are as follows:

(i) We formulate a novel constrained convex optimization problem under the network homogeneity assumption, in which the objective function is linear, but the optimal solution is dictated by a quasi-convex latency constraint. 
(ii) We solve this modified constrained optimization problem by using KKT conditions on the Lagrangian functions and derive closed-form expression for the cloudlet deployment cost.

(iii) We use these closed form expressions to illustrate that this optimal solution yields a tight lower bound for the framework proposed in [8] by making a performance comparison over an urban deployment area against $1 \mathrm{~ms}, 10 \mathrm{~ms}$, and $100 \mathrm{~ms}$ target latency values with an optical access network split ratio of $1: N, N \in\{4,8,16\}$.

(iv) We also perform a detailed parametric analysis to observe the changes in behavior of cost optimization framework against different network parameters like split ratio of TDM-PON, population density and quality-of-service (QoS) latency target.

The rest of this paper is organized as follows. Section 2 provides a brief review of some recent related works. Section 3 briefly discusses the TDM-PON based FiWi hybrid cloudlet placement network architecture. Section 4 formulates the constrained optimization problem for optimal cloudlet placement. Section 5 justifies the validity of the proposed analytical framework. Section 6 presents a detailed parametric analysis and explains the impacts of several network parameters on cloudlet cost-optimization frameworks. Finally, Section 7 concludes the paper by summarizing our primary findings.

\section{Related Work}

In this section, we explore some very recently published works on different aspects of cloudlet networks. The authors of [9-11] proposed computation offloading frameworks for energy saving in edge devices, assuming that cloudlets are already deployed over access networks. However, usually the user distribution under a typical wireless network deployment is very complicated. In addition, in crowded areas, cloudlets can be accessed by a large number of mobile users, whereas in sparsely populated areas only a few users will intend to access cloudlets. Thus, optimal placement of cloudlets over wireless access networks and placement of optimal number of VMs in cloudlets appeared to be essential research problems to several researchers because these aspects improve the cloudlet resource utilization significantly [12]. Note that, before the genesis of cloudlets and other edge-computing paradigms, researchers explored various VM migration techniques to optimize the power consumption in Network Function Virtualization (NFV) environments under a dynamic traffic scenario and became aware of its potential benefits $[13,14]$. The authors of [15] proposed a linear programming solution for computation offloading by considering the QoS requirements of mobile users while maximizing the revenue of service providers. The authors of $[7,16]$ proposed an edge-cloud network design framework that first determines where to install cloudlet facilities among available sites, and then assign sets of access points to cloudlets that supports VM orchestration as well satisfies service-level agreements. The authors of [17-21] proposed heuristic algorithms for static network planning to optimally place cloudlets over the existing wireless access network and followed by dynamic job request allocation to the cloudlets. The authors of $[22,23]$ focused on minimizing the energy consumption of the mobile devices while computation offloading to the cloudlets over wireless channels. Several researchers like [24-29] also took interest to design efficient dynamic resource allocation algorithms.

On the other hand, the authors of [30,31] identified that optical access networks can be useful in the next few decades to support edge computing technologies. In [32], the authors studied the performance of centralized and decentralized bandwidth allocation algorithms in a long-reach optical access network to investigate the feasibility of computation offloading to edge-computing servers and develop an analytical framework to validate against simulated results. The authors of [1] presented the implementation of a cloudlet framework for human-machine interactive applications with control server at the $\mathrm{CO}$ of a fiber-based access network. The authors of [33] presented the idea of cloud and cloudlet empowered FiWi-heterogeneous network architecture for LTE-A and designed a cloudlet-aware resource management algorithm that aims to reduce the offload latency and prolong mobile-devices' battery life. The authors of [34] designed a joint optimization algorithm of multiple jobs scheduling and investigated lightpath provisioning to minimize average completion time in fog 
computing micro data-centers elastic optical networks. Recently, in [35,36], we provided a high-level overview of latency-aware cloudlet placement frameworks over TDM-PON based FiWi environment. In [37], we proposed an MINLP based cost optimization framework for cloudlet placement in the field locations.

To the best of our knowledge, for the very first time, we proposed a cost-optimization framework for hybrid cloudlet placement in [8], where cloudlets are allowed to be installed in the field, RN and at $\mathrm{CO}$ locations subject to the capacity and latency constraints. In this work, we formulated an MINLP based cost-minimization problem and implemented using commercial solvers that use spatial branch-and-bound algorithms to find a global optimal solution. We applied this framework over stochastically generated $5 \mathrm{~km} \times 5 \mathrm{~km}$ urban, suburban, and rural areas with population densities 4000, 2500, 1500 persons $/ \mathrm{km}^{2}$, respectively, with QoS latency targets $1 \mathrm{~ms}, 10 \mathrm{~ms}$, and $100 \mathrm{~ms}$ to find optimal cloudlet placement locations. Through this exercise, we realized that, although this framework is capable of providing us the exact cloudlet placement locations, but it does not provide any general insight about cost-optimal cloudlet deployment strategies, depending on the underlying network scenario. Moreover, the solution algorithm does not scale very well with large data sets. These fundamental shortcomings motivated us to develop the analytical cost-optimization framework proposed in this paper. This analytical framework is based on network homogeneity assumption and performs an average analysis with a few network parameters e.g., split-ratio, population density, available bandwidth, and QoS latency requirements. Therefore, it can provide us a first-hand estimation of the deployment cost and several useful insights on the cost-optimal deployment strategies for any underlying network scenario, without any scalability issues.

\section{Hybrid Cloudlet Placement Architecture}

A static cloudlet placement framework considers the network status as static in time, i.e., it neither takes user mobility nor VM mobility into account and identifies optimal cloudlet locations over an existing access network infrastructure. This is essential to a cost-optimal network design framework for cloudlet placement and assignment of ONUs to cloudlets [16]. In this paper, we consider the same hybrid cloudlet placement framework over the TDM-PON based FiWi access network proposed in [8], as shown in Figure 1. Recall that we considered tree-and-branch network topology of TDM-PON with split-ratio $1: N$, where $N \in\{4,8,16\}$. Each ONU has an integrated wireless access point to serve multiple edge devices by wireless connection. The cloudlets can be suitably installed either in the field, at $\mathrm{RN}$ or at $\mathrm{CO}$ locations. Based on the assumption of network homogeneity, instead of analyzing the entire volume of the network, we can reduce our analysis to just a single TDM-PON based FiWi branch.

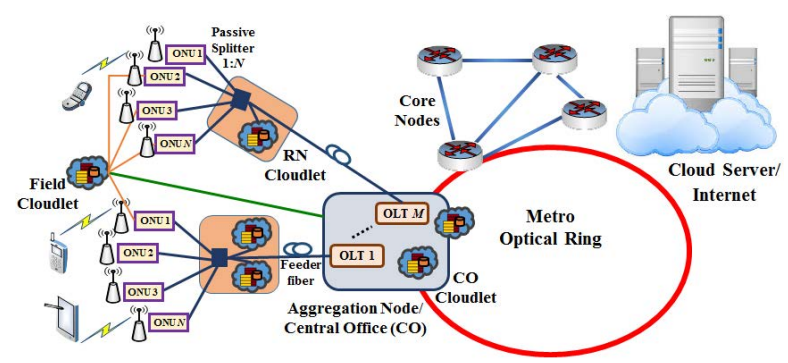

Figure 1. The hybrid cloudlet placement framework with cloudlets placed in the field, RN and CO of TDM-PON based FiWi network.

Each CO has multiple (e.g., 1,2, ., M) optical line terminals consisting of line cards and optical transceivers. We recall from [8] that ONUs can be connected to the field cloudlets via point-to-point fiber links (brown links). In that case, a new set of optical transceivers are installed both at the ONU and corresponding field cloudlet.

We consider the scope of installing cloudlets at RN locations along with the passive splitters. Each $R N$ cloudlet uses one or multiple new time-shared wavelengths for communication with ONUs in both 
uplink and downlink. In this case, a new set of optical transceivers are also required to be installed both at the ONU and RN cloudlet.

The CO cloudlets are installed at $\mathrm{CO}$ and hence are furthest from the ONUs. However, ONUs can use the spare bandwidth of the default uplink and downlink channels for communication with the $\mathrm{CO}$ cloudlets and hence no additional transceivers are required to be installed at ONUs or COs. However, in this work, we restrict our attention only to RN and CO cloudlets, as we showed in our previous work in [8] that these are the most cost-effective schemes for most practical cloudlet deployment.

\section{Optimal Cloudlet Placement Problem}

In this section, we present the system model and constrained convex optimization problem formulation to overcome the scalability issues observed with the MINLP formulation in [8]. Cloud servers are usually over-provisioned and possess huge computational and storage resources, hence can be assumed as $\mathrm{M} / \mathrm{M} / \infty$ queuing system [21]. On the other hand, the cloudlets contain a finite number of processors with application virtualization that uses a VM to provide an execution environment for the offloaded job requests [38]. Due to this reason, cloudlets can simultaneously perform different tasks and hence we consider that the cloudlet hardware is parallel processing enabled in this work [39]. From Google cluster-usage traces, it can be shown that job request arrival and their service times follow exponential distributions, and hence can be considered as Poisson processes [40]. Note that an $M / M / 1$ queue provides a lower-bound on processing latency as long as a single cloudlet has the aggregated processing rate of all the processors. Therefore, to compute the average processing latency, we model the cloudlets as $M / M / 1$ queueing systems [20].

We consider a single TDM-PON based FiWi branch where cloudlets can be installed at RN and CO locations, as shown in Figure 1. Table 1 briefly outlines the definitions of the required network parameters. In this work, we consider the maximum job request arrival rate as $\lambda_{\max }=p N$ (jobs/s), where $p$ denotes the maximum number of users each ONU can serve and $N$ denotes the split-ratio of each TDM-PON under network homogeneity assumption. The total transmission latency between a cloudlet and ONU is the sum of to-and-fro data transmission latency and average polling cycle latency, i.e., $D_{z}=\left(\frac{2 L_{z}}{v_{c}}+\frac{\delta_{z}}{2}\right), \forall z \in\{r, o\}$, where $L_{z}$ denotes the average length and $\delta_{z}$ denotes the average polling cycle latency between cloudlet at $z \in\{r, o\}$ and ONUs, and $v_{\mathcal{C}}$ denotes the velocity of light in optical fiber. We assume that the job request packets from ONUs and job response packets from cloudlets are highest priority packets in the network, and are processed within one polling cycle of the TDM-PON standard considered.

The decision variables are defined as follows: $x_{r}:=$ the fraction of total incoming workload assigned to the RN cloudlet, $x_{0}:=$ the fraction of total incoming workload assigned to the CO cloudlet, $b_{r}:=$ (binary variable) takes the value of 1 if an $\mathrm{RN}$ cloudlet is installed, $b_{0}:=$ (binary variable) takes the value of 1 if a CO cloudlet is installed, and $\mu:=$ total service rate of all the processors installed at the $\mathrm{RN}$ and CO cloudlets (jobs/s). A summary of all the decision variables are tabulated in Table 2 for convenience. All these variables are allowed to be only non-negative i.e., $\geq 0$. Thus, the objective function to minimize the overall cloudlet installation expenditures is given below:

$$
\min \left(\frac{\alpha}{\varphi} \mu+\xi_{r} b_{r}+\xi_{o} b_{o}\right)
$$

where the binary variables $b_{r}$ and $b_{o}$ follow the boundary constraints $x_{r} \leq b_{r} \leq\left(x_{r}+0.999\right)$ and $x_{0} \leq b_{0} \leq\left(x_{0}+0.999\right)$. This ensures that a cloudlet is installed at RN and CO only when $x_{r}>0$ and $x_{0}>0$. In objective function (1), the first component indicates the total cost of installing processors because $\varphi$ denotes the average number of jobs a single processor can simultaneously process and $\alpha$ denotes the cost of installing a single processor. As $\xi_{r}$ and $\xi_{o}$ denote the cost of new infrastructure installation for cloudlets, the second and third components indicate the cloudlet installation costs at $R N$ and at $\mathrm{CO}$ locations, respectively. However, due to the presence of $b_{r}$ and $b_{0}$, the problem still remains 
a mixed-integer programming problem and cannot be treated as a convex optimization problem. Therefore, to avoid this issue, we reformulate the problem. Instead of using $b_{r}$ and $b_{o}$ in the objective function, we use only a constant $\xi_{z}$ that indicates cloudlet installation cost in general and the modified objective function is:

$$
\min \left(\frac{\alpha}{\varphi} \mu+\xi_{z}\right)
$$

To ensure that all ONUs are served either by $\mathrm{RN}$ or $\mathrm{CO}$ cloudlet, we consider the constraint below:

$$
x_{r}+x_{o}=1 \quad \text { or, } \quad x_{0}=1-x_{r} .
$$

Again, to ensure the non-negativity of the decision variables, we also consider the following constraints:

$$
(\mu-p N) \geq 0 \text { and, } \quad 0 \leq x_{r} \leq 1 .
$$

The latency constraint that ensures that the overall system latency does not exceed $D_{Q o S}$ is given below:

$$
\begin{aligned}
& x_{r}\left\{\frac{1}{\mu x_{r}-p N x_{r}}+D_{r}+\frac{N \sigma_{u l}}{n_{\lambda} B W_{r}}+\frac{N \sigma_{d l}}{n_{\lambda} B W_{r}}\right\} \\
& \quad+x_{o}\left\{\frac{1}{\mu x_{o}-p N x_{o}}+D_{o}+\frac{N \sigma_{u l}}{B W_{o}-\beta_{u l}}+\frac{N \sigma_{d l}}{B W_{o}-\beta_{d l}}\right\} \leq D_{\mathrm{Qos}} .
\end{aligned}
$$

Table 1. Network optimization parameters.

\begin{tabular}{cl}
\hline Symbol & \multicolumn{1}{c}{ Definition } \\
\hline$\alpha$ & Cost of installing a single processor in a cloudlet \\
\hline$\varphi$ & Average number of jobs a single processor can simultaneously process \\
\hline$\xi_{z}$ & New infrastructure installation cost at location $z \in\{r, o\}$ \\
\hline$L_{z}$ & Average length between cloudlet at $z \in\{r, o\}$ and ONUs \\
\hline$\delta_{z}$ & Average polling cycle latency between cloudlet at $z \in\{r, o\}$ and ONUs \\
\hline$B W_{z}$ & $\begin{array}{l}\text { Bandwidth of the optical fiber link between cloudlets at } z \in\{r, o\} \text { and ONUs for both uplink } \\
\text { and downlink }\end{array}$ \\
\hline$n_{\lambda}$ & Number of wavelengths shared by ONUs to communicate to their corresponding RN cloudlet \\
\hline$D_{z}$ & Propagation latency between cloudlet at $z \in\{r, o\}$ and ONUs \\
\hline$D_{Q o S}$ & Maximum value of allowed QoS latency requirement \\
\hline$p$ & Maximum number of mobile users served by each ONU \\
\hline$N$ & Split-ratio of the passive splitter of the TDM-PON branches \\
\hline$\sigma_{u l}$ & Average number of bits an ONU sends to cloudlet at $z \in\{r, o\}$ for processing \\
\hline$\sigma_{d l}$ & Average number of bits an ONU receives from cloudlet at $z \in\{r, o\}$ after processing \\
\hline$\beta_{u l}$ & Background load in the uplink of the considered TDM PON \\
\hline$\beta_{d l}$ & Background load in the downlink of the considered TDM PON \\
\hline$v_{c}$ & Velocity of light within optical fiber $\left(2 \times 10^{8} \mathrm{~m} / \mathrm{s}\right)$ \\
\hline
\end{tabular}

Here, each of $x_{r}$ and $x_{o}$ are multiplied to compute the weighted average of the processing latency of the cloudlet, the propagation latency, and the total transmission latency, i.e., the latency to offload total bits for the job request from ONU to cloudlet, and the latency to receive total bits post-processing by ONU from cloudlet, respectively for RN and CO cloudlets. Note that $\frac{1}{\mu x_{r}-p N x_{r}}$ and $\frac{1}{\mu x_{0}-p N x_{0}}$ denote processing latency at RN and CO cloudlets, respectively. The terms $D_{r}$ and $D_{o}$ denote propagation 
latencies between ONUs and cloudlets at $\mathrm{RN}$ and $\mathrm{CO}$ locations, respectively. In addition to this, $\sigma_{u l}$ and $\sigma_{d l}$ denote the average number of bits an ONU sends to cloudlet and the average number of bits an ONU receives from cloudlet. These parameters are used to compute the total uplink and downlink transmission latency by the expressions $\left\{\frac{N \sigma_{u l}}{n_{\lambda} B W_{r}}+\frac{N \sigma_{d l}}{n_{\lambda} B W_{r}}\right\}$ and $\left\{\frac{N \sigma_{u l}}{B W_{o}-\beta_{u l}}+\frac{N \sigma_{d l}}{B W_{o}-\beta_{d l}}\right\}$, where $n_{\lambda}$ denotes the number of wavelengths ONUs share to communicate to their corresponding RN cloudlet, $B W_{r}$ and $B W_{o}$ denote the maximum available bandwidth (both in uplink and downlink) between ONUs and cloudlets at RN and $\mathrm{CO}$, respectively, and $\beta_{u l}$ and $\beta_{d l}$ denote the background load in the uplink and downlink, respectively, of the considered TDM-PON.

Table 2. Optimization decision variables.

\begin{tabular}{clc}
\hline Variable & \multicolumn{1}{c}{ Definition } & Type \\
\hline$b_{r}$ & Indicates if a cloudlet is installed at RN location & binary \\
$b_{o}$ & Indicates if a cloudlet is installed at CO location & binary \\
$x_{r}$ & The fraction of total incoming workload assigned to the RN cloudlet & real, continuous \\
$x_{o}$ & The fraction of total incoming workload assigned to the CO cloudlet & real, continuous \\
$\mu$ & Total service rate of all the processors installed in all cloudlets (jobs/s) & real, continuous \\
\hline
\end{tabular}

Now, using the constraint (3), moving denominator terms to numerator and rearranging all terms, we rewrite constraint (5) as below:

$$
2+\left\{(A-B) x_{r}+\left(B-D_{\mathrm{QoS}}\right)\right\}(\mu-p N) \leq 0,
$$

where

$$
\begin{aligned}
& A=D_{r}+\frac{N \sigma_{u l}}{n_{\lambda} B W_{r}}+\frac{N \sigma_{d l}}{n_{\lambda} B W_{r}}, \\
& B=D_{o}+\frac{N \sigma_{u l}}{B W_{o}-\beta_{u l}}+\frac{N \sigma_{d l}}{B W_{o}-\beta_{d l}} .
\end{aligned}
$$

Therefore, the reformulated optimization problem can be written as a standard optimization problem as follows:

$$
\begin{aligned}
\boldsymbol{P}: \quad \min _{\mu, x_{r}}\left(\frac{\alpha}{\varphi} \mu+\xi_{z}\right) \\
\text { subject to } \quad(\mu-p N) \geq 0, \\
0 \leq x_{r} \leq 1, \\
2+\left\{(A-B) x_{r}+\left(B-D_{\mathrm{QoS}}\right)\right\}(\mu-p N) \leq 0 .
\end{aligned}
$$

Proposition 1. The function $f\left(x_{r}, \mu\right)=2+\left\{(A-B) x_{r}+\left(B-D_{Q o S}\right)\right\}(\mu-p N)$ is non-convex/concave but quasi-convex in nature.

Figure 2 shows the contours of $f\left(x_{r}, \mu\right)=0$ as a function of the variables $x_{r}$ and $\mu$ against different values of split-ratio $N$ and $D_{Q o S}=1 \mathrm{~ms}$. By carefully observing the contour plots, we can understand the quasi-convex nature of the curves. A more rigorous proof is given in the Appendix A. 


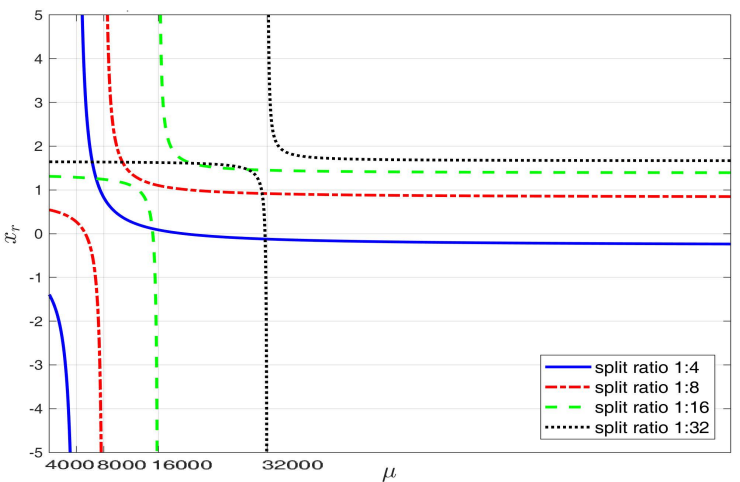

Figure 2. Contour plots of $f\left(x_{r}, \mu\right)=0$ against different values of split-ratio $N \in\{4,8,16\}$ and $D_{Q o S}=1 \mathrm{~ms}$.

Theorem 1. To find the global optimal solution of the constrained optimization problem with objective function (2) and constraints (3)-(5), the first-order KKT conditions are necessary and sufficient.

Proof. This theorem can be proven in a straightforward manner. We observe that the objective function (2) and the constraint (4) are linear and the Proposition 1 shows that the constraint (5) is quasi-concave. Therefore, if there exists at least one Lagrange multiplier that satisfy the KKT conditions, then any local optimum is the global optimum for this problem. Hence, the first-order KKT (necessary) conditions are the sufficient conditions for optimality [41].

Therefore, we write the Lagrangian function for the objective function (2) with constraints (3)-(5) as follows:

$$
\begin{aligned}
\mathcal{L}\left(x_{r}, \mu ; \lambda\right)= & \frac{\alpha}{\varphi} \mu+\xi_{z}-\lambda_{1}\left(1-x_{r}\right)-\lambda_{2} x_{r}-\lambda_{3}(\mu-p N) \\
& +\lambda_{4}\left[2+\left\{(A-B) x_{r}+\left(B-D_{Q o S}\right)\right\}(\mu-p N)\right],
\end{aligned}
$$

and derive the first-order KKT conditions for optimality from the Lagrangian function (A1) as follows:

$$
\begin{aligned}
& \frac{\partial \mathcal{L}}{\partial \mu}=\frac{\alpha}{\varphi}-\lambda_{3}+\lambda_{4}\left\{(A-B) x_{r}+\left(B-D_{Q o S}\right)\right\}=0, \\
& \frac{\partial \mathcal{L}}{\partial x_{r}}=\lambda_{1}-\lambda_{2}+\lambda_{4}(A-B)(\mu-p N)=0 \\
& \frac{\partial \mathcal{L}}{\partial \lambda_{1}}=-x_{r}, \frac{\partial \mathcal{L}}{\partial \lambda_{2}}=-\left(1-x_{r}\right), \frac{\partial \mathcal{L}}{\partial \lambda_{3}}=-(\mu-p N), \\
& \frac{\partial \mathcal{L}}{\partial \lambda_{4}}=2+\left\{(A-B) x_{r}+\left(B-D_{Q o S}\right)\right\}(\mu-p N)=0 .
\end{aligned}
$$

Theorem 2. The cloudlet cost optimization framework installs all cloudlets either at $R N$ locations when $A<B$, $A<D_{\text {QoS }}$, or at CO locations when $B<A, B<D_{\text {QoS }}$. However, when both $A<D_{\text {QoS }}$ and $B<D_{Q o S}$ are satisfied together, then the cloudlet installation location is chosen based on $\xi_{r}$ and $\xi_{0}$.

Proof. From the KKT conditions (10) and (11), we observe that if $A<B, A<D_{\mathrm{QoS}}$, then $x_{r}-1=0$ or, $x_{r}=1$, and $\lambda_{1} \neq 0$. This implies that $x_{r} \neq 0$ and $\lambda_{2}=0$. To keep the processing time finite, we must have $(\mu-p N) \geq 0$, i.e., $(\mu-p N) \neq 0$ and hence $\lambda_{3}=0$. However, the latency constraint can be tight, which implies that $2+\left\{(A-B) x_{r}+\left(B-D_{Q o S}\right)\right\}(\mu-p N)=0$ and $\lambda_{4} \neq 0$. Hence, we find the optimal solutions as below: 


$$
\begin{aligned}
& \mu^{*}=\left[p N+\frac{2}{D_{\mathrm{QoS}}-A}\right], \\
& \lambda_{1}^{*}=\frac{2 \alpha(B-A)}{\varphi\left(D_{\mathrm{QOS}}-A\right)^{2}}, \quad \lambda_{4}^{*}=\frac{\alpha}{\varphi\left(D_{\mathrm{QOS}}-A\right)} .
\end{aligned}
$$

Therefore, we calculate the optimal cloudlet installation cost as follows, where all cloudlets are installed at $\mathrm{RN}$ locations $\left(x_{r}^{*}=1, x_{o}^{*}=0\right)$ :

$$
\mathbb{C}_{1}=\frac{\alpha}{\varphi}\left[p N+\frac{2}{D_{\mathrm{QoS}}-A}\right]+\xi_{r}
$$

Again, note that if $B<A, B<D_{\mathrm{Q} o S}$, then $x_{r}-1 \neq 0$ and $\lambda_{1}=0$, which implies that $x_{r}=0$ and $\lambda_{2} \neq 0$. We have $(\mu-p N) \neq 0$ and $\lambda_{3}=0$, same as before. In addition, $2+\left\{(A-B) x_{r}+(B-\right.$ $\left.\left.D_{\mathrm{QoS}}\right)\right\}(\mu-p N)=0$ and $\lambda_{4} \neq 0$. Thus, the optimal solutions are computed as follows:

$$
\begin{aligned}
& \bar{\mu}^{*}=\left[p N+\frac{2}{D_{\mathrm{QoS}}-B}\right], \\
& \bar{\lambda}_{2}^{*}=\frac{2 \alpha(A-B)}{\varphi\left(D_{\mathrm{QoS}}-B\right)^{2}}, \quad \bar{\lambda}_{4}^{*}=\frac{\alpha}{\varphi\left(D_{\mathrm{QoS}}-B\right)} .
\end{aligned}
$$

These values yield the following optimal cloudlet installation cost, where all cloudlets are installed at $\mathrm{CO}$ locations $\left(x_{r}^{*}=0, x_{0}^{*}=1\right)$ :

$$
\mathbb{C}_{2}=\frac{\alpha}{\varphi}\left[p N+\frac{2}{D_{\mathrm{QoS}}-B}\right]+\xi_{0} .
$$

When both $A<D_{\mathrm{QoS}}$ and $B<D_{\mathrm{QoS}}$ are satisfied together, we can freely install cloudlets at either of $\mathrm{RN}$ and $\mathrm{CO}$ locations, but both of these do not provide the cost optimal solution simultaneously, if $\xi_{r} \neq \xi_{o}$ (we consider $\xi_{r}>\xi_{o}$ ). Therefore, in this case, we should compare both $\mathbb{C}_{1}$ and $\mathbb{C}_{2}$ first, and then choose to install cloudlets at $\mathrm{RN}$ locations if $\mathbb{C}_{1}<\mathbb{C}_{2}$, or $\mathrm{CO}$ locations if $\mathbb{C}_{1}>\mathbb{C}_{2}$.

It is interesting to note that when $A=B$, then $0 \leq x_{r} \leq 1$ holds but $x_{r}^{*}$ does not have any unique optimal value. In this situation, we have $\lambda_{1}=\lambda_{2}=\lambda_{3}=0$ and the following optimal values:

$$
\begin{aligned}
& \hat{\mu}^{*}=\left[p N+\frac{2}{D_{\mathrm{QoS}}-A}\right]=\left[p N+\frac{2}{D_{\mathrm{QoS}}-B}\right], \\
& \hat{\lambda}_{4}^{*}=\frac{\alpha}{\varphi\left(D_{\mathrm{QoS}}-B\right)}=\frac{\alpha}{\varphi\left(D_{\mathrm{Q} o S}-A\right)} .
\end{aligned}
$$

Nonetheless, even in this situation, it is best to install cloudlets only at one location because installing cloudlets at two locations will lead only to a higher cloudlet installation cost as follows:

$$
\mathbb{C}_{3}=\frac{\alpha}{\varphi}\left[p N+\frac{2}{D_{Q o S}-B}\right]+\xi_{r}+\xi_{o}
$$

Clearly, the above cases show that optimal cloudlet placement framework should install cloudlets only at either of $\mathrm{RN}$ and $\mathrm{CO}$ locations, because $\mathbb{C}_{3}>\mathbb{C}_{1}$ and $\mathbb{C}_{3}>\mathbb{C}_{2}$, always.

\section{Framework Validation}

In this section, we make a performance comparison of the analytical framework derived in Section 3 with the complete MINLP framework proposed in [8]. To achieve this goal, we proceed in two steps. Firstly, we stochastically generate multiple instances of network data over a circular deployment area, i.e., ONU locations, RN locations, and CO location. We evaluate the MINLP based 
framework designed in [8] on these data and obtain an empirical average of the results. Secondly, we derive a theoretical model for the circular network deployment area such that the circular area can be reduced to the smallest circular wedge under the network homogeneity assumption. We apply the suitable expressions derived in Section 3 on this circular wedge and scale up the results to obtain similar results as obtained from the first step.

\subsection{Random Dataset Generation and MINLP Evaluation}

We consider a circular area of diameter $4 \mathrm{~km}$ and average population density of 4000 persons $/ \mathrm{km}^{2}$, similar to a typical Australian urban area [42]. As we do not have access to any real data of ONU locations from any network service providers, we use Poisson-point process over this circular area to generate random ONU locations, which provides almost realistic distribution of ONU locations [43]. Each ONU can serve a maximum of 1000 users, hence for the average population density 4000 persons $/ \mathrm{km}^{2}$, we need to install $4 \mathrm{ONUs} / \mathrm{km}^{2}$ on an average. We consider 10G-PON standard such that $10 \mathrm{Gbps}$ data rate is available in both uplink and downlink of the access network. In this analysis, we consider TDM-PON split-ratio $1: N, N \in\{4,8,16\}$ and $D_{\text {QoS }}$ values of $1 \mathrm{~ms}$, $10 \mathrm{~ms}$, and $100 \mathrm{~ms}$ and generate multiple instances of network deployment data. For the sake of some geometrical advantages, we consider that the CO of all TDM-PON branches are co-located at the centre of the circular area. To evaluate the MINLP framework, we use the A Modeling Language for Mathematical Programming (AMPL) platform and the open-source solver COUENNE package [https:/ / projects.coin-or.org/Couenne].

\subsection{Homogeneous Circular Area for Analytical Framework}

Now, we reduce the entire circular area to circular wedges by considering the cloudlet deployment network homogeneous. With $\Gamma$ ONUs $/ \mathrm{km}^{2}$, a total $\left\lceil\Gamma \pi R^{2}\right\rceil$ number of ONUs can exist in a circular area of radius $R$ and hence $\Omega=\left\lceil\Gamma \pi R^{2} / N\right\rceil$ TDM-PONs of split-ratio $N$ are sufficient to serve all the ONUs. Based on this, we reduce the entire circular area as a combination of $\Omega$ identical circular wedges inscribing a central angle, $2 \theta=2 \pi / \Omega$. For example, in Figure 3, we show a case for $\Gamma=4 \mathrm{ONUs} / \mathrm{km}^{2}$, $R=2 \mathrm{~km}$, and $N=8$, there are $\Omega=7$ identical circular wedges with $2 \theta=2 \pi / 7$. Due to the network homogeneity assumption, we can consider that the RNs are located at the centroids of the circular wedges. The coordinates of the centroid of a circular wedge with radius $R$ and spanned from $-\theta$ to $\theta$ are $(\bar{x}, \bar{y})=\left(\frac{2 R}{3 \theta} \sin \theta, 0\right)$ [https://en.wikipedia.org/wiki/List_of_centroids], and the distance between $\mathrm{CO}$ and $\mathrm{RN}$ is, $L_{c r}=\left(\frac{2 R}{3 \theta} \sin \theta\right) \mathrm{km}$. The average distance between ONU and RN is derived as follows:

$$
L_{r o}=\frac{1}{\theta R^{2}}\left[\int_{-\theta}^{\theta} \int_{0}^{\bar{x}}(\bar{x}-x) r d r d \theta+\int_{-\theta}^{\theta} \int_{\bar{x}}^{R}(x-\bar{x}) r d r d \theta\right] .
$$

Evaluating the double integrals in (21) by putting $x=r \cos \theta$ and $\bar{x}=2 R \sin \theta / 3 \theta$, we obtain the final expression as follows:

$$
L_{r o}=\frac{16 R}{27 \theta^{3}} \sin ^{3} \theta\left(1-\frac{2}{3 \theta} \sin \theta\right) .
$$

As we consider the tree-and-branch topology of TDM-PON architecture [44], the total length between an ONU and CO is the sum of the distances from CO to RN and RN to ONU. Therefore, the average distance between an ONU and $\mathrm{CO}\left(L_{c o}\right)$ within the circular wedge is $L_{c o}=L_{c r}+L_{r o}$. The key advantage of reducing the entire circular area to circular wedges is that we can apply the analytical formulae derived in Section 3 to compute the average cloudlet deployment cost with a single TDM-PON based FiWi branch and then scale up the results by multiplying with factor $\Omega$ to calculate the total cloudlet deployment cost over the entire circular area. 


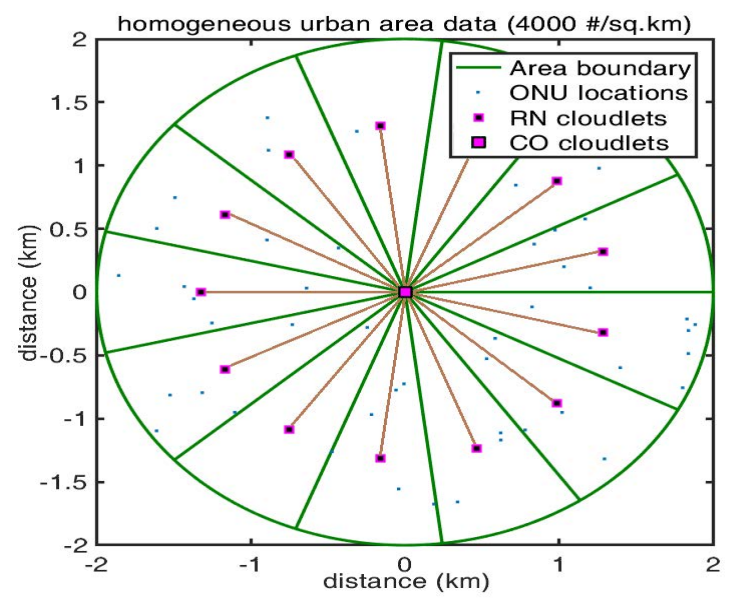

Figure 3. An example homogeneous network with ONU locations (blue dots), RN locations (black squares), and CO locations (pink squares) with split-ratio 1:8 over an urban population area with 4000 people $/ \mathrm{km}^{2}$ (only the feeder fibers are shown).

\subsection{Performance Comparison among the Frameworks}

While evaluating either of the expressions for cost values $\mathbb{C}_{1}, \mathbb{C}_{2}$, and $\mathbb{C}_{3}$, as required, we need to validate that our convex optimization problem formulation produces an equivalent result to that of the original MINLP problem with objective function (1). Hence, we evaluate both the frameworks for a single TDM-PON based FiWi branch and scale up the results for the entire network. In addition to this, we need to show that the scaled up results are close enough to the results obtained by using the MINLP framework on the complete dataset and hence we compare these cost values with the empirical average of cost values obtained by evaluating the MINLP framework in [8] on the multiple instances of randomly generated complete datasets. The same values of all the network parameters are considered as in [8] to maintain a consistency in our analysis. The normalized cost of installing a processor is $\alpha=1$ [https://www.lenovo.com/gb/en/workstations/p-series/ThinkStation-P900/p/33TS3TPP900], the normalized costs of installing a cloudlet at $\mathrm{RN}$ is $\xi_{r}=6$ and at $\mathrm{CO}$ is $\xi_{0}=5$ [https:/ /www.lenovo.com/ $\mathrm{gb} / \mathrm{en} /$ workstations/p-series/ThinkStation-P900/p/33TS3TPP900], $n_{\lambda}=1$ wavelength of data rate $B W_{r}=10 \mathrm{Gbps}$ is shared among all $N$ ONUs to communicate with $\mathrm{RN}$ cloudlet, the data rate of default communication link between $\mathrm{ONU}$ and $\mathrm{CO}$ is $B W_{o}=10 \mathrm{Gbps}$, the approximate downlink and uplink background loads are $\beta_{d l}=7 \mathrm{Gbps}$ and $\beta_{u l}=5 \mathrm{Gbps}$, respectively [45], each ONU serves $p=1000$ users, each cloudlet processor supports $\varphi=2500 \mathrm{VMs}$ [16], average number of bits an ONU sends to a cloudlet as job requests in uplink $\sigma_{u l}=1 \mathrm{MB}$ and average number of bits an ONU receives from a cloudlet as response of the job requests in downlink $\sigma_{d l}=50 \mathrm{~KB}$ [4]. The average polling cycle latency parameters are $\delta_{r}=\delta_{0}=0.5 \mathrm{msec}$ [46].

In Figure 4, we present the comparison among normalized cloudlet deployment cost/100 users over the considered circular deployment area by using the MINLP framework on the complete dataset, by using the MINLP framework on a single TDM-PON branch and scaling up the results, and scaling up the results from the newly proposed analytical framework. In this analysis, we consider TDM-PON split-ratio $1: N, N \in\{4,8,16\}$ and $D_{\mathrm{QoS}}$ values of $1 \mathrm{~ms}, 10 \mathrm{~ms}$, and $100 \mathrm{~ms}$. The optimal cost values obtained by using analytical framework is expected to be lower bounds to the actual MINLP framework. The workload assignment fractions $x_{r}$ and $x_{o}$ are not present in the objective function, but only in the latency constraint; hence, this newly designed framework puts more importance towards meeting the latency constraint. Moreover, this analytical framework is better in terms of estimating cloudlet deployment cost under low-latency requirement than simply converting the integral decision variables of the framework in [8] into continuous decision variables. 


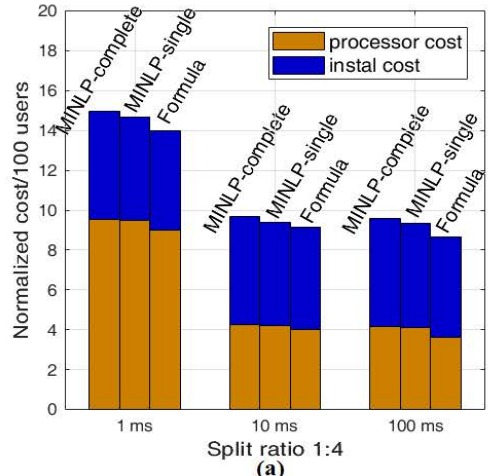

(a)
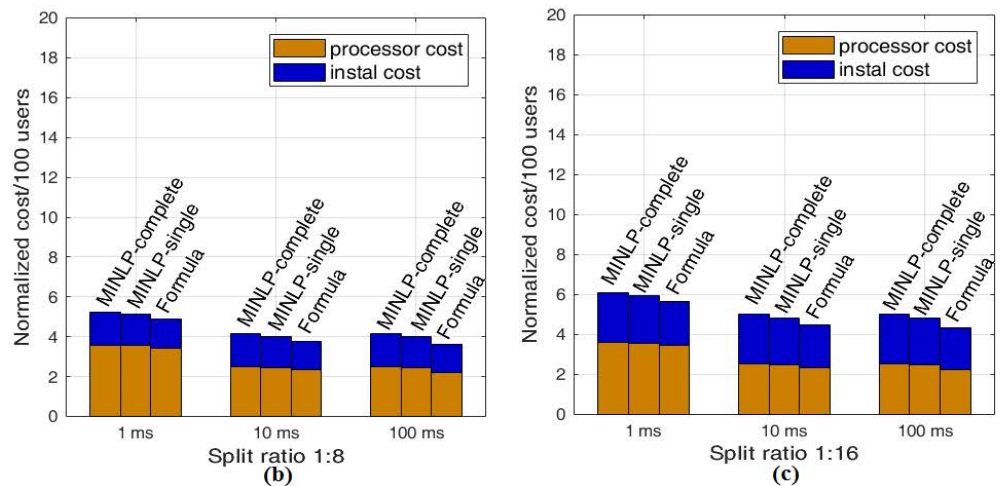

(c)

Figure 4. Comparison of normalized cloudlet deployment cost/100 users with population density 4000 persons $/ \mathrm{km}^{2}$ computed using the modified analytical formula designed in this paper with the complete MINLP framework proposed in [8] and MINLP on a single TDM-PON FiWi branch against $D_{\text {QoS }}$ values of $1 \mathrm{~ms}, 10 \mathrm{~ms}$, and $100 \mathrm{~ms}$ with (a) split-ratio 1:4; (b) split-ratio 1:8; and (c) split-ratio 1:16.

\section{Results and Discussion}

In this section, we perform a parametric analysis and discuss some of the key insights obtained about the behaviour of the analytical cost optimization framework against variations in values of different network parameters.

In Figure 5, we present the workload distribution among the $\mathrm{RN}$ and $\mathrm{CO}$ cloudlets against TDM-PON split-ratio over a circular area with diameter $=4 \mathrm{~km}$, population density $=4000$ people $/ \mathrm{km}^{2}$, and $D_{\mathrm{QoS}}=1 \mathrm{~ms}$. With smaller split-ratio values, e.g., 1:4 and 1:8, the total workload can be processed by CO cloudlets, hence $x_{o}=1$ and $x_{r}=0$. Thus, all cloudlets are installed at CO locations and none at $\mathrm{RN}$ locations. However, for a higher split-ratio of 1:16, there appears a bandwidth crunch for the ONUs to access the CO cloudlets. Therefore, under this condition, the cost optimization framework chooses to install cloudlet at $\mathrm{RN}$ locations over $\mathrm{CO}$ locations and the same is observed from the plot as well.

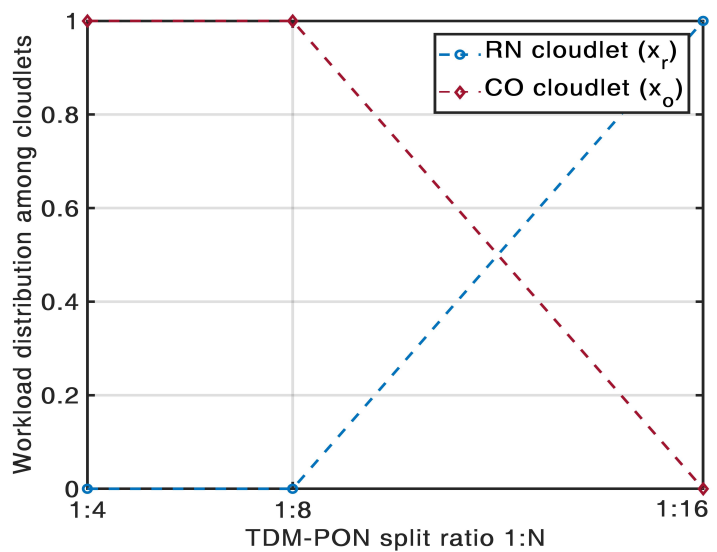

Figure 5. Workload distribution among $\mathrm{RN}$ and $\mathrm{CO}$ cloudlets against TDM-PON split ratio for a circular area with diameter $=4 \mathrm{~km}$, population density $=4000$ people $/ \mathrm{km}^{2}$, and $D_{Q o S}=1 \mathrm{~ms}$.

In Figure 6, we show the variation of normalized cloudlet deployment cost/100 users against TDM-PON split-ratio $1: N$, where $N \in\{4,8,16\}$ with $D_{\mathrm{QoS}}=1 \mathrm{~ms}$. We vary the population density from 1000 to 4000 people $/ \mathrm{km}^{2}$ in steps of 1000 . From these plots, we observe that the normalized cloudlet deployment cost decreases with increase in split-ratio from 1:4 to 1:8, because a lower number of TDM-PON branches are required to handle the workload and all cloudlets are installed at same $\mathrm{CO}$ locations. However, there is a slight increase in the normalized cost as the split ratio is further increased to 1:16. This happens because the framework chooses to install all cloudlets at RN locations, where the cost of cloudlet installation is higher than that of $\mathrm{CO}$ locations. We further observe that the 
normalized cost is higher for lower population density with smaller split-ratio, e.g., with split-ratio 1:4, normalized cost for 1000 people $/ \mathrm{km}^{2}$ is higher than that of 4000 people $/ \mathrm{km}^{2}$. However, this behaviour is reversed with a higher split-ratio 1:16.

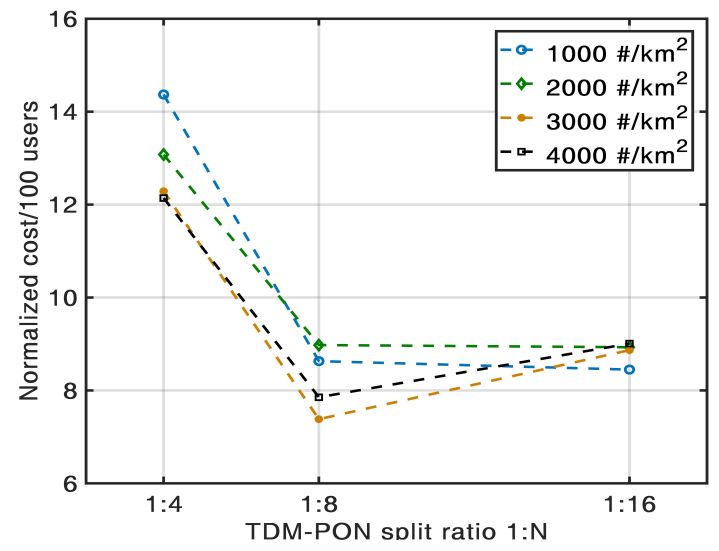

Figure 6. Variation of normalized cloudlet deployment cost/100 users against TDM-PON split-ratio $1: N$ for a circular area with diameter $=4 \mathrm{~km}$ and $D_{\mathrm{QoS}}=1 \mathrm{~ms}$.

Figure 7 shows the variation of normalized cloudlet deployment cost/100 users against population density of the considered circular cloudlet deployment area. We vary the TDM-PON split-ratio $1: N$ where $N \in\{4,8,16\}$ and $D_{\mathrm{QoS}}=1 \mathrm{~ms}$. We observe that the overall cloudlet installation cost increases with increasing population density, but the normalized cost/100 users slowly decreases for a particular split-ratio. Whenever a new TDM-PON is added, we observe a sudden jump in the normalized cost. In general, the normalized cost is minimum with split-ratio 1:8 and maximum with split-ratio 1:4.

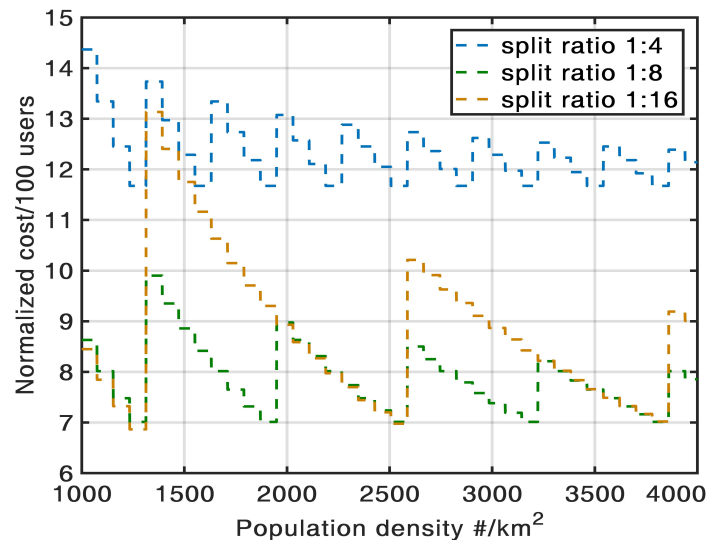

Figure 7. Variation of normalized cloudlet deployment cost/100 users against population density of the cloudlet deployment circular area with diameter $=4 \mathrm{~km}$ and $D_{Q o S}=1 \mathrm{~ms}$.

Next, Figure 8 shows the variation of normalized cloudlet deployment cost/100 users with population density 4000 people $/ \mathrm{km}^{2}$ against TDM-PON split-ratio $1: N$ where $N \in\{4,8,16\}$ while varying $D_{\mathrm{Q} o s}$. These plots follow similar trends as we observed in Figure 6 . The normalized cloudlet deployment cost first decreases as split-ratio increases from 1:4 to 1:8, and then the normalized cost slightly increases again for the split-ratio 1:16. Note that the normalized costs for a stringent QoS requirement of $D_{Q o S}=1 \mathrm{~ms}$ are highest and gradually continue to become lower as the QoS requirement is relaxed to $D_{\mathrm{QoS}}=100 \mathrm{~ms}$. This behaviour is observed due to the fact that a higher amount of computational resources (i.e., processors) are required to meet a stringent $D_{\mathrm{QoS}}=1 \mathrm{~ms}$ than a lenient $D_{\text {QoS }}=100 \mathrm{~ms}$. 


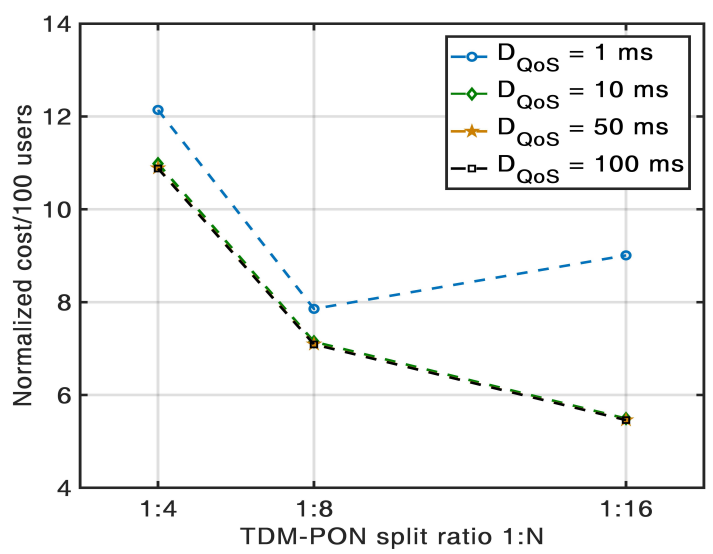

Figure 8. Variation of normalized cloudlet deployment cost/100 users against TDM-PON split-ratio $1: N$ for a circular area with diameter $=4 \mathrm{~km}$ and population density $=4000$ people $/ \mathrm{km}^{2}$.

Finally, in Figure 9, we show a comparison of normalized cloudlet deployment cost/100 users against $D_{\mathrm{QoS}}=1 \mathrm{~ms}$ with homogeneous and non-homogeneous network deployment. We choose the same circular area with a diameter of $4 \mathrm{~km}$ and population density 4000 people $/ \mathrm{km}^{2}$. To simulate non-homogeneity, we choose a bunch of TDM-PONs and randomly vary their split-ratios such that their mean is equal to the corresponding homogeneous split-ratio $1: N$ where $N \in\{4,8,16\}$. For example, for a homogeneous split-ratio of $1: 8$, we uniformly choose split-ratios of the TDM-PONs from the interval $[6,10]$ such that their mean is 8 , and so on. We observe from our simulated results that the performance of the non-homogeneous framework is not worse than $5 \%$ of that with the homogeneous framework.

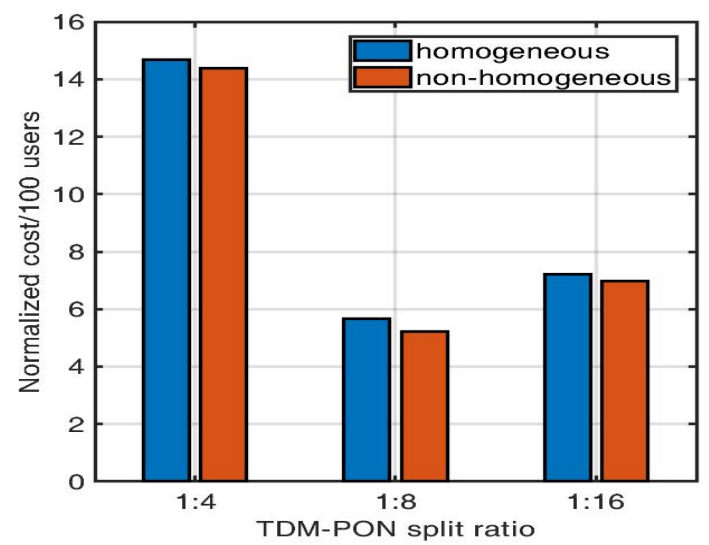

Figure 9. Comparison of normalized cloudlet deployment cost/100 users with homogeneous and non-homogeneous network over a circular area with diameter $=4 \mathrm{~km}$, population density $=$ 4000 people $/ \mathrm{km}^{2}$, and $D_{Q o S}=1 \mathrm{~ms}$.

\section{Conclusions}

In this paper, we have designed an analytical cost optimization framework that provides a tight lower bound of the optimal deployment cost for the integer/mixed-integer programming based cost-optimization frameworks on a hybrid cloudlet placement architecture, where cloudlets are optimally placed either at RN or at CO locations of a TDM-PON based FiWi access network. As we have formulated a constrained convex optimization problem which is analytically solvable by using KKT conditions, this framework can efficiently operate on large datasets without requiring any heuristic algorithms. This analytical framework not only resolves the scalability issues of the MINLP based framework proposed in [8], but also provides a tight lower bound of the integer programming based framework. We have considered multiple instances of a circular area of diameter 
$4 \mathrm{~km}$ with population density 4000 people $/ \mathrm{km}^{2}$ to evaluate the MINLP framework and the analytical framework proposed in this paper. Thus, we have verified that the optimal costs obtained using the analytical frameworks lie within $10 \%$ of the optimal costs obtained by using the MINLP framework. We have also performed a parametric analysis to show the impact on cloudlet deployment cost of various network parameters like user density, network architecture, TDM-PON split-ratio and QoS requirements. Overall, our proposed analytical framework provides valuable guidance to network and service operators on a good first-hand estimate of cloudlet deployment cost. Results from our analysis also clearly point to the fact that, in the evaluation of optimal cost, the latency constraint plays a dominant role.

Author Contributions: Writing-original draft preparation, S.M.; formal analysis, S.M.; software, S.M.; supervision, G.D. and E.W.; writing—-review and editing, G.D. and E.W.

Funding: This research received no external funding.

Conflicts of Interest: The authors declare no conflict of interest.

\section{Appendix A. Proof of Proposition 1}

Proof. We compute the first-order partial derivatives of $f\left(x_{r}, \mu\right)$ with respect to $x_{r}$ and $\mu$ as follows:

$$
\begin{aligned}
\frac{\partial f}{\partial x_{r}} & =(A-B)(\mu-p N), \\
\frac{\partial f}{\partial \mu} & =\left\{(A-B) x_{r}+\left(B-D_{Q o S}\right)\right\} .
\end{aligned}
$$

To analyze the convexity of the function $f\left(x_{r}, \mu\right)$, we evaluate the Hessian matrix [41] as follows:

$$
\begin{gathered}
\mathcal{H}=\left[\begin{array}{cc}
\frac{\partial^{2} f}{\partial x_{r}^{2}} & \frac{\partial^{2} f}{\partial x_{r} \partial \mu} \\
\frac{\partial^{2} f}{\partial \mu \partial x_{r}} & \frac{\partial^{2} f}{\partial \mu^{2}}
\end{array}\right]=\left[\begin{array}{cc}
0 & (A-B) \\
(A-B) & 0
\end{array}\right], \\
\operatorname{det}(\mathcal{H})=-(B-A)^{2} .
\end{gathered}
$$

Clearly, $\mathcal{H}$ is not positive semi-definite as $\operatorname{det}(\mathcal{H})<0$, and hence $f\left(x_{r}, \mu\right)$ is a non-convex/concave function [41]. Nonetheless, a function may posses local maxima and minima. Thus, we evaluate the bordered Hessian matrix [41] for the function $f\left(x_{r}, \mu\right)$ as follows:

$$
\begin{gathered}
\mathcal{H}_{B}=\left[\begin{array}{ccc}
0 & \frac{\partial f}{\partial x_{r}} & \frac{\partial f}{\partial \mu} \\
\frac{\partial f}{\partial x_{r}} & \frac{\partial^{2} f}{\partial x_{r}^{2}} & \frac{\partial^{2} f}{\partial x_{r} \partial \mu} \\
\frac{\partial f}{\partial \mu} & \frac{\partial^{2} f}{\partial \mu \partial x_{r}} & \frac{\partial^{2} f}{\partial \mu^{2}}
\end{array}\right], \\
\operatorname{det}\left(\mathcal{H}_{B}\right)=-2(B-A)^{2}(\mu-p N)\left\{(B-A) x_{r}-\left(B-D_{Q o S}\right)\right\} .
\end{gathered}
$$

We can easily show that, when the constraint (6) holds, then $\operatorname{det}\left(\mathcal{H}_{B}\right)<0$. Therefore, we can consider $f\left(x_{r}, \mu\right)$ as a quasi-convex function, as $\mathcal{H}_{B}$ is of odd-order $(=3)$ [41].

\section{References}

1. Wong, E.; Dias, M.P.I.; Ruan, L. Predictive Resource Allocation for Tactile Internet Capable Passive Optical LANs. IEEE J. Lightw. Technol. 2017, 35, 2629-2641. [CrossRef]

2. Satyanarayanan, M.; Bahl, P.; Caceres, R.; Davies, N. The Case for VM-Based Cloudlets in Mobile Computing. IEEE Pervas. Comput. 2009, 8, 14-23. [CrossRef]

3. Satyanarayanan, M. The Role of Cloudlets in Hostile Environments. In Proceedings of the Fourth ACM Workshop on Mobile Cloud Computing and Services (MCS '13), Taipei, Taiwan, 25-28 June 2013; ACM: New York, NY, USA, 2013; pp. 1-2. [CrossRef] 
4. Ha, K.; Pillai, P.; Lewis, G.; Simanta, S.; Clinch, S.; Davies, N.; Satyanarayanan, M. The Impact of Mobile Multimedia Applications on Data Center Consolidation. In Proceedings of the 2013 IEEE International Conference on Cloud Engineering (IC2E '13), Redwood City, CA, USA, 25-27 March 2013; IEEE Computer Society: Washington, DC, USA, 2013; pp. 166-176. [CrossRef]

5. Rimal, B.P.; Van, D.P.; Maier, M. Mobile Edge Computing Empowered Fiber-Wireless Access Networks in the 5G Era. IEEE Commun. Mag. 2017, 55, 192-200. [CrossRef]

6. Aldaya, I.; Del-Valle-Soto, C.; Campuzano, G.; Giacoumidis, E.; González, R.; Castañón, G. Photonic millimeter-wave bridge for multi-Gbps passive optical networks. Phys. Commun. 2018, 28, 138 - 146. [CrossRef]

7. Ceselli, A.; Premoli, M.; Secci, S. Mobile Edge Cloud Network Design Optimization. IEEE/ACM Trans. Netw. 2017, 25, 1818-1831. doi:10.1109/TNET.2017.2652850. [CrossRef]

8. Mondal, S.; Das, G.; Wong, E. CCOMPASSION: A Hybrid Cloudlet Placement Framework over Passive Optical Access Networks. In Proceedings of the INFOCOM 2018-2018 IEEE Conference on Computer Communications, Honolulu, HI, USA, 16-19 April 2018, pp. 1-9.

9. Chun, B.G.; Ihm, S.; Maniatis, P.; Naik, M.; Patti, A. CloneCloud: Elastic Execution Between Mobile Device and Cloud. In Proceedings of the Sixth Conference on Computer Systems (EuroSys '11), Salzburg, Austria, 10-13 April 2011; ACM: New York, NY, USA, 2011; pp. 301-314. [CrossRef]

10. Cuervo, E.; Balasubramanian, A.; Cho, D.K.; Wolman, A.; Saroiu, S.; Chandra, R.; Bahl, P. MAUI: Making Smartphones Last Longer with Code Offload. In Proceedings of the 8th International Conference on Mobile Systems (MobiSys '10), Applications, and Services, San Francisco, CA, USA, 15-18 June 2010; ACM: New York, NY, USA, 2010; pp. 49-62. [CrossRef]

11. Kemp, R.; Palmer, N.; Kielmann, T.; Bal, H. Cuckoo: A Computation Offloading Framework for Smartphones. In Mobile Computing, Applications, and Services; Gris, M., Yang, G., Eds.; Springer: Berlin/Heidelberg, Germany, 2012; pp. 59-79.

12. Li, K.; Nabrzyski, J. Virtual Machine Placement in Cloudlet Mesh. J. Commun. Netw. 2018, 20, $266-278$. [CrossRef]

13. Eramo, V.; Tosti, A.; Miucci, E. Server Resource Dimensioning and Routing of Service Function Chain in NFV Network Architectures. J. Electrical Comput. Eng. 2016, 2016, 7139852. [CrossRef]

14. Eramo, V.; Miucci, E.; Ammar, M. Study of Migration Policies in Energy-Aware Virtual Router Networks. IEEE Commun. Lett. 2014, 18, 1919-1922. [CrossRef]

15. Hoang, D.T.; Niyato, D.; Wang, P. Optimal Admission Control Policy for Mobile Cloud Computing Hotspot with Cloudlet. In Proceedings of the 2012 IEEE Wireless Communications and Networking Conference (WCNC), Shanghai, China, 1-4 April 2012; pp. 3145-3149. [CrossRef]

16. Ceselli, A.; Premoli, M.; Secci, S. Cloudlet Network Design Optimization. In Proceedings of the 2015 IFIP Networking Conference (IFIP Networking), Toulouse, France, 20-22 May 2015; pp. 1-9. [CrossRef]

17. Xu, Z.; Liang, W.; Xu, W.; Jia, M.; Guo, S. Efficient Algorithms for Capacitated Cloudlet Placements. IEEE Trans. Parallel Distrib. Syst. 2016, 27, 2866-2880. [CrossRef]

18. Fan, Q.; Ansari, N. Cost Aware Cloudlet Placement for Big Data Processing at the Edge. In Proceedings of the 2017 IEEE International Conference on Communications (ICC), Paris, France, 21-25 May 2017; pp. 1-6. [CrossRef]

19. Sun, X.; Ansari, N. PRIMAL: PRofIt Maximization Avatar pLacement for Mobile Edge Computing. arXiv 2015, arXiv:1510.04340.

20. Sun, X.; Ansari, N. Latency Aware Workload Offloading in the Cloudlet Network. IEEE Commun. Lett. 2017, 21, 1481-1484. [CrossRef]

21. Jia, M.; Cao, J.; Liang, W. Optimal Cloudlet Placement and User to Cloudlet Allocation in Wireless Metropolitan Area Networks. IEEE Trans. Cloud Comput. 2015, 5, 725-737. [CrossRef]

22. Gai, K.; Qiu, M.; Zhao, H.; Tao, L.; Zong, Z. Dynamic Energy-aware Cloudlet-based Mobile Cloud Computing Model for Green Computing. J. Netw. Comput. Appl. 2016, 59, 46-54. [CrossRef]

23. Gai, K.; Qiu, M.; Zhao, H. Energy-aware Task Assignment for Mobile Cyber-enabled Applications in Heterogeneous Cloud Computing. J. Parallel Distrib. Comput. 2018, 111, 126-135. [CrossRef]

24. Gai, K.; Qiu, M. Optimal Resource Allocation Using Reinforcement Learning for IoT Content-centric Services. Appl. Soft Comput. 2018, 70, 12 - 21. [CrossRef] 
25. Liu, Y.; Lee, M.J.; Zheng, Y. Adaptive Multi-Resource Allocation for Cloudlet-Based Mobile Cloud Computing System. IEEE Trans. Mob. Comput. 2016, 15, 2398-2410. [CrossRef]

26. Fan, Q.; Ansari, N. Workload Allocation in Hierarchical Cloudlet Networks. IEEE Commun. Lett. $2018, P P, 1$. [CrossRef]

27. Zhao, X.; Zhang, Y.; Wei, L.; Lu, M. Computational and Communication Resource Allocation for Mobile Cooperative Cloudlet Computing Systems. In Proceedings of the 2015 International Conference on Wireless Communications Signal Processing (WCSP), Nanjing, China, 15-17 October 2015; pp. 1-5. [CrossRef]

28. Liu, L.; Fan, Q. Resource Allocation Optimization Based on Mixed Integer Linear Programming in the Multi-Cloudlet Environment. IEEE Access 2018, 6, 24533-24542. [CrossRef]

29. Jin, A.; Song, W.; Zhuang, W. Auction-Based Resource Allocation for Sharing Cloudlets in Mobile Cloud Computing. IEEE Trans. Emerg. Top. Comput. 2018, 6, 45-57. [CrossRef]

30. Newaz, S.H.S.; Mohammed, A.F.Y.; Lee, G.M.; Choi, J.K. Energy efficient and latency aware TDM-PON for local customer internetworking. In Proceedings of the 2015 IFIP/IEEE International Symposium on Integrated Network Management (IM), Ottawa, ON, Canada, 11-15 May 2015; pp. 1184-1189. [CrossRef]

31. Newaz, S.H.S.; binti Haji Suhaili, W.S.; Lee, G.M.; Uddin, M.R.; Mohammed, A.F.Y.; Choi, J.K. Towards realizing the importance of placing fog computing facilities at the central office of a PON. In Proceedings of the 2017 19th International Conference on Advanced Communication Technology (ICACT), Bongpyeong, Korea, 19-22 February 2017; pp. 152-157. [CrossRef]

32. Helmy, A.H.; Nayak, A. Integrating Fog With Long-Reach PONs From a Dynamic Bandwidth Allocation Perspective. IEEE J. Lightw. Technol. 2018, 36, 5276-5284. [CrossRef]

33. Rimal, B.P.; Van, D.P.; Maier, M. Cloudlet Enhanced Fiber-Wireless Access Networks for Mobile-Edge Computing. IEEE Trans. Wirel. Commun. 2017, 16, 3601-3618. [CrossRef]

34. Liu, Z.; Zhang, J.; Li, Y.; Bai, L.; Ji, Y. Joint Jobs Scheduling and Lightpath Provisioning in Fog Computing Micro Datacenter Networks. IEEE/OSA J. Opt. Commun. Netw. 2018, 10, 152-163. [CrossRef]

35. Wong, E.; Mondal, S.; Das, G. Latency-aware Optimisation Framework for Cloudlet Placement. In Proceedings of the 2017 19th International Conference on Transparent Optical Networks (ICTON), Girona, Spain, 2-6 July 2017; pp. 1-2. [CrossRef]

36. Mondal, S.; Das, G.; Wong, E. Support of Low Latency Applications through Hybrid Cost-Optimised Cloudlet Placement. In Proceedings of the 2018 20th International Conference on Transparent Optical Networks (ICTON), Bucharest, Romania, 1-5 July 2018; pp. 1-4.

37. Mondal, S.; Das, G.; Wong, E. A Novel Cost Optimization Framework for Multi-Cloudlet Environment over Optical Access Networks. In Proceedings of the GLOBECOM 2017-2017 IEEE Global Communications Conference, Singapore, 4-8 December 2017, pp. 1-7. [CrossRef]

38. Flinn, J.; Park, S.; Satyanarayanan, M. Balancing performance, energy, and quality in pervasive computing. In Proceedings of the 22nd International Conference on Distributed Computing Systems, Vienna, Austria, 2-5 July 2002; pp. 217-226. [CrossRef]

39. Goldberg, R.P. Survey of Virtual Machine Research. Computer 1974, 7, 34-45. [CrossRef]

40. Jiang, C.; Chen, Y.; Wang, Q.; Liu, K.J.R. Data-Driven Stochastic Scheduling and Dynamic Auction in IaaS. In Proceedings of the 2015 IEEE Global Communications Conference (GLOBECOM), San Diego, CA, USA, 6-10 December 2015; pp. 1-6. [CrossRef]

41. Miller, R.E. Optimization: Foundations and Applications; John Wiley \& Sons, Inc.: Hoboken, NJ, USA, 1999.

42. Spencer, A.; Gill, J.; Schmahmann, L. Urban or Suburban? Examining The Density of Australian Cities in a Global Context. In Proceedings of the the State of Australian Cities Conference 2015, Gold Coast, QLD, Austrilia, 9-11 December 2015; pp. 9-11.

43. Matthews, S.; Parker, D.M. Progress in Spatial Demography. Demogr. Res. 2013, S13, 271-312. [CrossRef]

44. Wong, E. Next-Generation Broadband Access Networks and Technologies. IEEE J. Lightw. Technol. 2012, 30, 597-608. [CrossRef] 
45. Wong, E.; Machuca, C.M.; Wosinska, L. Survivable Hybrid Passive Optical Converged Network Architectures Based on Reflective Monitoring. IEEE J. Lightw. Technol. 2016, 34, 4317-4328. [CrossRef]

46. Skubic, B.; Chen, J.; Ahmed, J.; Wosinska, L.; Mukherjee, B. A Comparison of Dynamic Bandwidth Allocation for EPON, GPON, and Next-generation TDM PON. IEEE Commun. Mag. 2009, 47, S40-S48. [CrossRef]

(C) 2019 by the authors. Licensee MDPI, Basel, Switzerland. This article is an open access article distributed under the terms and conditions of the Creative Commons Attribution (CC BY) license (http:/ / creativecommons.org/licenses/by/4.0/). 